This item was submitted to Loughborough's Research Repository by the author.

Items in Figshare are protected by copyright, with all rights reserved, unless otherwise indicated.

\title{
A comparison of the flow fields generated for spark and controlled auto- ignition
}

PLEASE CITE THE PUBLISHED VERSION

http://dx.doi.org/10.4271/2003-01-1798

\section{PUBLISHER}

(C) Society of Automotive Engineers of Japan

\section{VERSION}

VoR (Version of Record)

\section{PUBLISHER STATEMENT}

This work is made available according to the conditions of the Creative Commons Attribution-NonCommercialNoDerivatives 4.0 International (CC BY-NC-ND 4.0) licence. Full details of this licence are available at: https://creativecommons.org/licenses/by-nc-nd/4.0/.

\section{LICENCE}

CC BY-NC-ND 4.0

\section{REPOSITORY RECORD}

Pitcher, Graham, James W.G. Turner, Graham Wigley, and Rui Chen. 2014. "A Comparison of the Flow Fields Generated for Spark and Controlled Auto-ignition”. figshare. https://hdl.handle.net/2134/15621. 
JSAE 20030223

SAE 2003-01-1798

\title{
A Comparison of the Flow Fields Generated for Spark and Controlled Auto-ignition
}

\author{
Graham Pitcher, James Turner \\ Lotus Engineering
}

Graham Wigley, Rui Chen

Loughborough University

2003 JSAE/SAE International Spring Fuels \& Lubricants Meeting

Yokohama, Japan

May 19-22, 2003 
Notice about photocopy

In order to photocopy any works from this publication, you or your organization must obtain permission from the following organization which you has been delegated for copyright for clearance by the copyright owner of this publication.

Except in the U.S.A.

Japan Academic Association for Copyright Clearance (JAACC)

6-41 Akasaka 9-chome, Minato-ku, Tokyo 107-0052 Japan

TEL: 81-3-3475-5618 Fax: 81-3-3475-5619 E-mail:naka-atsu@mju.biglobe.ne.jp

In the U.S.A.

Copyright Clearance Center, Inc.

222 Rosewood Drive, Danvers, MA 01923 U.S.A.

Phone: 1-978-750-8400 Fax:1-978-750-4744

Copyright @ 2003 Society of Automotive Engineers of Japan, Inc. 
JSAE 20030223

SAE 2003-01-1798

\title{
A Comparison of the Flow Fields Generated for Spark and Controlled Auto-ignition
}

\author{
Graham Pitcher and James Turner \\ Lotus Engineering, UK \\ Graham Wigley and Rui Chen \\ Loughborough University, UK
}

Copyright @ 2003 Society of Automotive Engineers of Japan, Inc.

\begin{abstract}
Valve timing strategies aimed at producing internal exhaust gas re-circulation in a conventional spark ignition, SI, engine have recently demonstrated the ability to initiate controlled auto-ignition, CAl. Essentially the exhaust valves close early, to trap a quantity of hot exhaust gases in-cylinder, and the fresh air-fuel charge is induced late into the cylinder and then mixing takes place. As a logical first step to understanding the fluid mechanics, the effects of the standard and modified valve timings on the in-cylinder flow fields under motored conditions were investigated. Laser Doppler anemometry has been applied to an optical engine that replicates the engine geometry and different valve cam timings. The cycle averaged time history mean and RMS velocity profiles for the axial and radial velocity components in three axial planes were measured throughout the inlet and compression stroke. The turbulent mixing for the two cases are described in terms of the flow field maps of the velocity vectors, vorticity and turbulence kinetic energy and the integrated tumble ratio as a function of crankangle.
\end{abstract}

\section{INTRODUCTION}

Controlled auto-ignition (CAI) is a part-load gasoline combustion process that is known to offer massive reductions in NOx emissions. Furthermore, its inherent stability on a cycle-to-cycle basis means combustion variance decreases, leading to increases in fuel economy and decreases in pollutant formation. Several valve timing strategies [1-4] have recently demonstrated the ability to initiate CAl. This also gives the ability to run the engine throttleless which has a direct contribution, through reductions in pumping losses, to the observed fuel economy improvements. One strategy adopted to achieve CAl is by:- (a) closing the exhaust valves early, to trap a known quantity of hot exhaust gases in-cylinder, (b) opening the inlet valve at a correspondingly late time, so as to equalise in-cylinder trapped gas pressures with those of inlet manifold pressures, and (c) the normal induction of fresh fuel-air charge then ensues.

With this system the fresh air-fuel charge is induced into the cylinder containing residual hot exhaust gases. The air/fuel mixing and gas exchange process will therefore be different to those of a conventional SI engine. Ando and Kuwahara [5], describing predominantly spark ignition systems, also comment that the limitation of combustion techniques such as CAl is the ability to initiate ignition and control the timing. Furthermore, only through the use of continuously variable valve timing (CVVT) can the control of residual exhaust gas as a parameter for ignition control be exploited. These comments support previous work on CAl in a single cylinder research engine equipped with active valve timing (AVT) $[2,3]$. The AVT engine was used to switch from conventional SI to CAl combustion to determine the optimum cam profiles.

A detailed understanding of the gas mixing and exchange processes between the fresh air-fuel charge and the residual exhaust gases trapped incylinder is obviously needed. As a first logical step to understanding the fluid mechanics, the effects of the modified valve timing on the in-cylinder flow fields under motored conditions were investigated. It is argued that the charge mixing in each case is still dominated by the in-cylinder geometry and the inlet flow field. This work therefore concentrated on measuring the in-cylinder flow field development by utilising two different sets of camshafts, one with conventional profiles and the other with modified profiles to simulate the gas mixing with internal EGR. 


\section{EXPERIMENTAL}

\section{OPTICALLY ACCESSED ENGINE}

The optical engine is a single cylinder research engine, incorporating a fused silica liner and sapphire piston crown to provide optical access, shown in Figure 1. Like the AVT engine it was based on one cylinder of a 1.8L, 4 cylinder engine and has a bore of $80.5 \mathrm{~mm}$ and a stroke of $88.2 \mathrm{~mm}$. The inlet valves have a diameter of $31 \mathrm{~mm}$ and are inclined at $68^{\circ}$ to the cylinder head face in the pent roof combustion chamber. The engine has been designed specifically for laser diagnostic research and has both primary and secondary balance shafts to allow for high-speed operation, up to 5000rpm. The head is based on a production design and maintains the exact geometry. A carbon fibre piston ring running in the optical liner maintains the correct compression pressures.

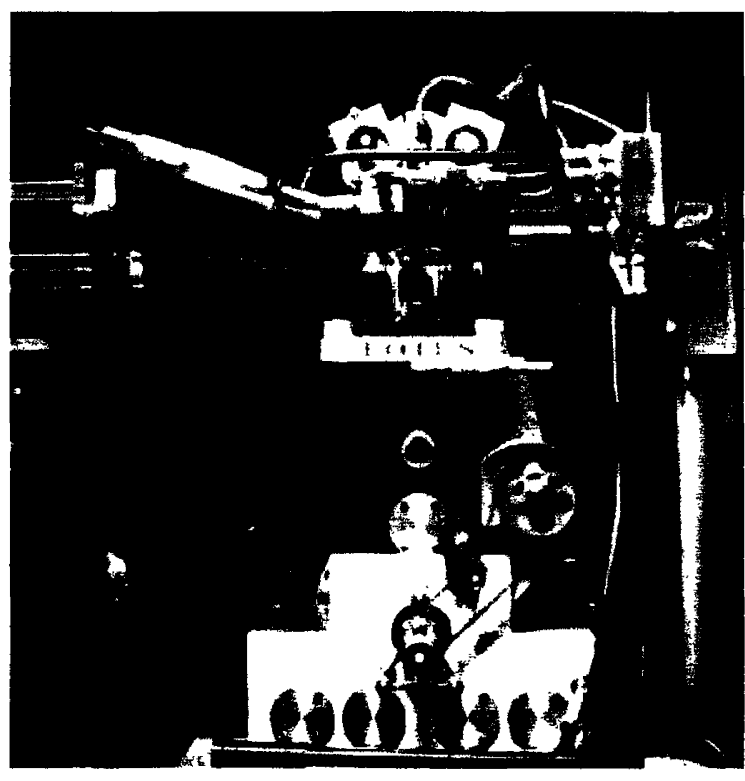

Figure 1. $0.45 \mathrm{~L}$ single cylinder optically accessed engine.

The engine was motored at 1500 rpm throughout for the measurements reported here. Essential details of the engine and camshaft profiles can be found in Table 1.

In the work presented here on the optical engine the cam profiles used were slightly different to the optimum determined using the AVT engine. The reason for this originates from attempting to design mechanical cams to emulate the work of the AVT system. Erroneous cam profile following can result when the valve tip and cam faces depart from contact under high acceleration. The easiest solution to this problem was to reduce the valve lift from the standard $6 \mathrm{~mm}$ to $3.6 \mathrm{~mm}$. To examine the consequences of the reduced valve lift profiles the AVT engine was run with both the $3.6 \mathrm{~mm}$ and the standard $6 \mathrm{~mm}$ lift. Emissions data from the TEXCEL system and compression pressure at top dead centre showed that the overall effect on these parameters was small and therefore the difference in valve lift was assumed to be minimal. This experiment will be further described with possible explanations in the results and discussion section.

Table 1: Single cylinder engine details.

\begin{tabular}{|c|c|}
\hline Bore & $80.5 \mathrm{~mm}$ \\
\hline Stroke & $88.2 \mathrm{~mm}$ \\
\hline Compression Ratio & $10.5: 1$ \\
\hline Max speed & $5000 \mathrm{rpm}$ \\
\hline Number of valves & 4 \\
\hline $\begin{array}{c}\text { Peak Compression } \\
\text { Pressure }\end{array}$ & $23 \mathrm{bar}$ \\
\hline \multicolumn{2}{|c|}{ Conventional Cam Timing } \\
\hline Inlet Valve Opening & 705 degrees \\
\hline Inlet Valve Closing & 235 degrees \\
\hline Exhaust Valve Opening & 504 degrees \\
\hline Exhaust Valve Closing & 28 degrees \\
\hline Maximum Valve Lift & 8.5 mm (Inlet) \\
\hline \multicolumn{2}{|c|}{ Modified Valve Timing } \\
\hline Inlet Valve Opening & 63 degrees \\
\hline Inlet Valve Closing & 247 degrees \\
\hline Exhaust Valve Opening & 493 degrees \\
\hline Exhaust Valve Closing & 677 degrees \\
\hline Maximum Valve Lift & 3.6 mm \\
\hline
\end{tabular}

\section{LASER ANEMOMETER SYSTEM}

The two component laser Doppler anemometer (LDA) system is described in detail in [6], with the only differences being the receiver optics configured for back scatter light collection and the inclusion of a two dimensional traverse system for computer controlled scanning of the measurement volume inside the glass liner. As all the measurements were made through the curved liner wall, alignment for coincidence of the two orthogonal measurement volumes would have been time consuming to achieve over the whole of the measurement mesh. Therefore, it was only used as a single component system with the two measured components being collected sequentially.

The inlet manifold was seeded with a mist of silicone oil generated by a medical nebuliser to act as light scattering centres for the LDA system. The mean droplet size was 3 to 5 microns. The LDA data were processed with a Dantec Enhanced PDA processor operated in 'velocity only' mode. For two component measurements this processor demands near perfect coincidence for the two signals and, as that could not be guaranteed without considerable effort, was the main reason for performing sequential single component measurements.

The number of single component velocity samples acquired at each point in a measurement plane varied from 30000 close to the head down to 15000 close to the cylinder bottom. As seeding levels were kept relatively sparse, to minimise window soiling and to ensure that data were collected over a sufficient number of engine cycles, data arrival rates were generally low and did not allow a cycle resolved analysis of the flow data. However, the well defined tumble flow structure exhibited small cycle to cycle variations particularly with the CAl cam profiles. 


\section{MEASUREMENTS}

Cycle averaged time history mean and RMS velocity profiles for the axial and radial velocity components have been made in three axial planes in the cylinder throughout the inlet and compression stroke. The measurement planes are shown in figure 2 with, from top to bottom; the plane of symmetry on a diameter between the inlet valves; the orthogonal diameter parallel to the pent roof of the combustion chamber and; a chord through the centre of one inlet and exhaust valve.

For each plane the measurements started at $10 \mathrm{~mm}$ below the head face and $5 \mathrm{~mm}$ from the cylinder inner wall. The axial scan was in $10 \mathrm{~mm}$ increments and the horizontal scan in $5 \mathrm{~mm}$ increments. Data were not collected on the cylinder centre-line. The crank angle resolved measurements were obtained between top dead centre valve overlap, and top dead centre compression. An optical encoder, 3600 pulses per revolution, attached to the cam gave a resolution of 0.2 crank degrees for the arrival time history of the discrete velocity data samples.

\section{RESULTS AND DISCUSSION}

The two individual components of crankangle resolved mean and RMS velocity profiles have been combined to allow the presentation of planar incylinder vector flow fields and contour plots of the turbulence kinetic energy and vorticity.

\section{DATA PROCESSING}

The initial processing of the discrete velocity samples was to bin the data obtained over many engine cycles into consecutive 4 degree crankangle windows and calculate the cycle averaged mean and RMS velocity for each time bin. The mean axial and radial velocities for each bin have then been combined for all positions in each plane to produce time varying vector flow fields to show how the inlet flow develops and changes throughout the engine cycle.

The complementary RMS values have been processed to generate planar time dependent turbulence kinetic energy, TKE, levels as defined by the following equation where $u^{\prime}, v^{\prime}$ and $w^{\prime}$ are the fluctuating velocity components in the orthogonal directions $x, y$ and $z$ :

$$
T K E=\frac{u^{\prime 2}+v^{\prime 2}+w^{\prime 2}}{2}
$$

As only two components of the RMS velocity were available from the measurements the third component, for this calculation, has been taken as the mean of the two measured components. These results are displayed in the form of planar contour plots.
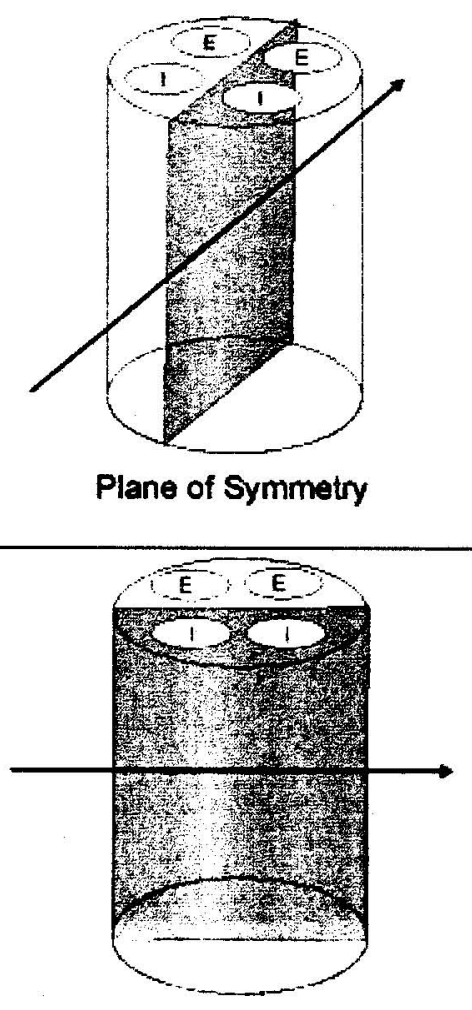

Plane Across Pent Roof

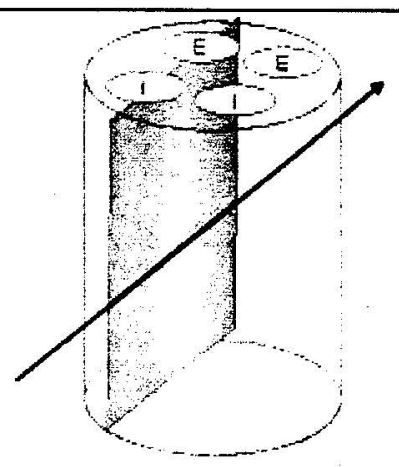

Plane Under Valves

Figure 2: Measurement planes 1,2 and 3 (I and $E$ signify inlet and exhaust valves respectively).

The mean velocity vectors in the plane have been further processed to gain greater insight into, and to quantify, the flow structures. The curl of the vector field can be used to define the vorticity of the flow which, when plotted as contours, allows visualisation of large re-circulation zones.

$$
C u r l=\zeta=\left[\frac{\delta v}{\delta x}-\frac{\delta u}{\delta y}\right]
$$

The mean vorticity for all positions in the plane can be used to calculate the tumble ratio of the flow field. For a solid body rotation the vorticity is twice the value of the angular velocity, so, for the tumble ratio calculation the mean vorticity value has been halved, $[7,8]$. 


$$
\begin{gathered}
\text { Tumble }- \text { ratio }=\frac{\sum_{n=1}^{N} \zeta_{n}}{2 N \omega} \\
\text { N --- Number of vorticity values } \\
\omega \text {--- Engine speed in radians/second }
\end{gathered}
$$

By the nature of the point-wise LDA measurements, the curl has to be calculated in a discrete form. For the tumble ratio calculations, a value has been found at the mid point of each set of four vectors on a rectangle in the plane. The tumble ratio is then based on the mean of all vorticity values. For the vorticity contour plots, where the emphasis is on the large scale structure, the vorticity has been calculated with a coarser mesh. One point in the centre of each set of nine vectors has been chosen, which highlights the rotational structures with less contribution from local three dimensional structures, which may also affect the curl value.

\section{VECTOR FIELDS}

Although planar vector flow fields for all crankangle sectors are available only flow fields in the symmetry plane for selected crank angles are to be presented. Only four flow field pairs are shown in Figure 3 with the left of each pair produced by the conventional cams while those on the right were obtained for the cams modified for CAl. It must be remembered that these are only 'snapshots' of the planar flow field while the discussion is based on the full time history of the flow fields. The crankangle sectors chosen for presentation correspond to:- (1) 74 degrees maximum tumble ratio for the conventional cams, (2) 114 degrees - maximum tumble ratio for the modified cams, (3) 154 degrees - fully developed flow for the modified cams and (4) 178 degrees - representing the time when the influence from the inlet flow is diminishing. In this symmetry plane, the mixing of the flow from the two inlet valves generates a three dimensional pattern which manifests itself whenever vectors at adjacent points appear inconsistent.

The most obvious difference between the two flows in figure 3 is the time delay imposed on the inlet flow field by the late opening of the inlet valves with the modified cams. Both flows show the development of the expected tumble motion, the inlet flow is directed towards the cylinder walls opposite which then turns to be guided by the piston face towards the inlet side of the cylinder. However, there is a relatively strong counter rotating vortex present with the modified cams, shown at 154 and 178 degrees in the lower half of the cylinder to the right of the centre line, which is absent from the other field. The other major difference between the two fields is the persistence of the developed flow field for the modified cams up to, and beyond, bottom dead centre, whereas for the conventional cams the flow is decaying rapidly by this time and becoming dominated by the piston motion.

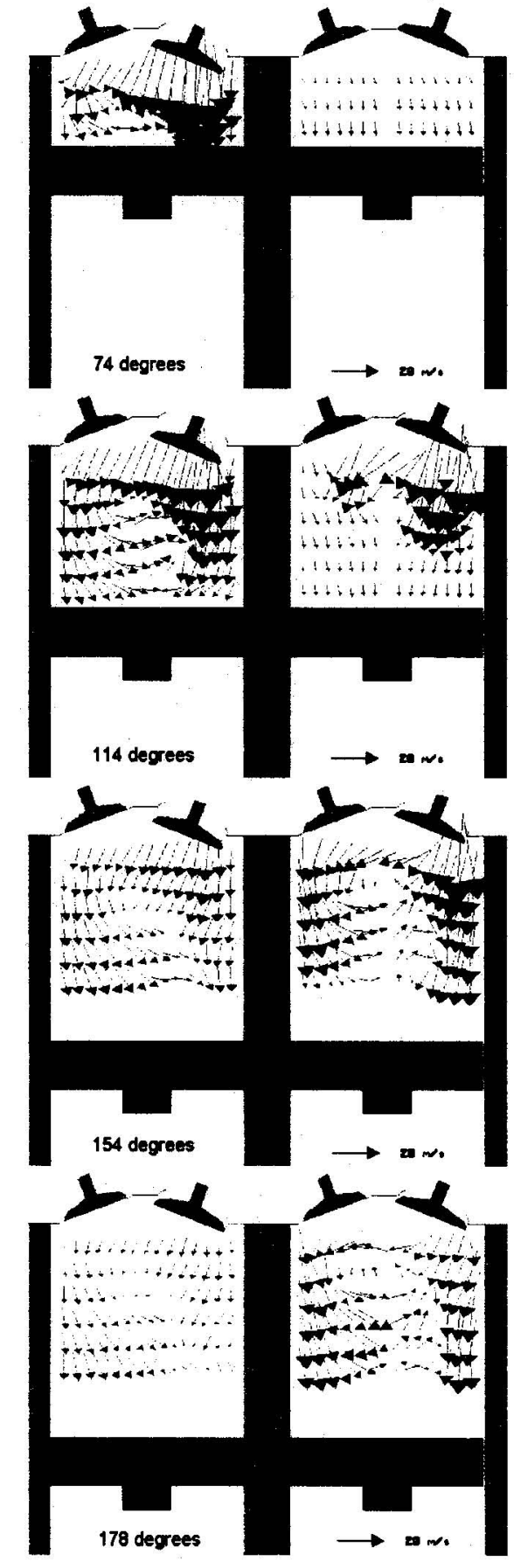

Figure 3. Vector Flow Fields in the Symmetry Plane

Similar comments can be made about the flows in plane 3, the chord through the inlet and exhaust valve centres. Here both cams were shown to generate two main counter rotating vortices, one under each valve. The vortex under the exhaust valve, rotating in the tumble direction, stays close to the head throughout the inlet stroke, while that under the inlet valve follows the piston down the cylinder The modified cams indicated the formation of a third vortex late in the stroke on the side of the exhaust valve but close to the piston. 
The flow fieids in plane 2, parallel to the pent roof, show counter rotating vortices either side of the cylinder centre due to the interaction of the inlet flow with the curved cylinder wall. A high degree of symmetry in the flow structure about the centreline was found for both cam profiles. The major difference between the two cams is the location of the highest inlet air velocities. For the conventional cams, the highest velocities are observed across the cylinder centre, while those for the modified cams are either side, at the cylinder walls.

\section{VORTICITY}

The vorticity plots are presented in the form of contour plots, which help to show the major recirculation zones or vortices. In general the modified cams show evidence of more, smaller vortices being generated, and in the later part of the cycle, these vortices have a much greater rotational velocity. This is believed to be a phenomenon generated purely by the engine geometry, due to the much smaller valve lift for these cams causing greater inlet velocity through the valve gap. An example of these phenomena are shown in figure 4 , at a time close to bottom dead centre, where the vortices for the conventional cams, upper figure, are diminishing, but those for the modified cams, lower figure, still retain significant angular velocity. The presence of an additional vortex can also be seen for the modified cams at coordinates $-7.5,45 \mathrm{~mm}$.

The degree of symmetry of the vortex structures in plane 2 for the modified cams is shown in figure 5 . Even though there is residual gas in the cylinder for the modified cams, both configurations show similar penetration of the vortices towards the bottom of the cylinder.

\section{TUMBLE RATIO}

The tumble ratio gives a global number against crankangle as an indication of bulk flow motion. This allows a simple comparison of integral differences between the flow fields for the two modes of operation.

The tumble ratios in the symmetry plane are shown in Figure 6, with the corresponding inlet valve lift profiles for the two configurations. In both cases the highest magnitude of tumble is seen during the inlet valve opening period, with the conventional cams exhibiting much higher tumble ratios. The modified cams have a second lower peak just after maximum valve lift at 160 degrees. By bottom dead centre the two curves are starting to merge, as any influence from the inlet jets diminishes as the valves close.
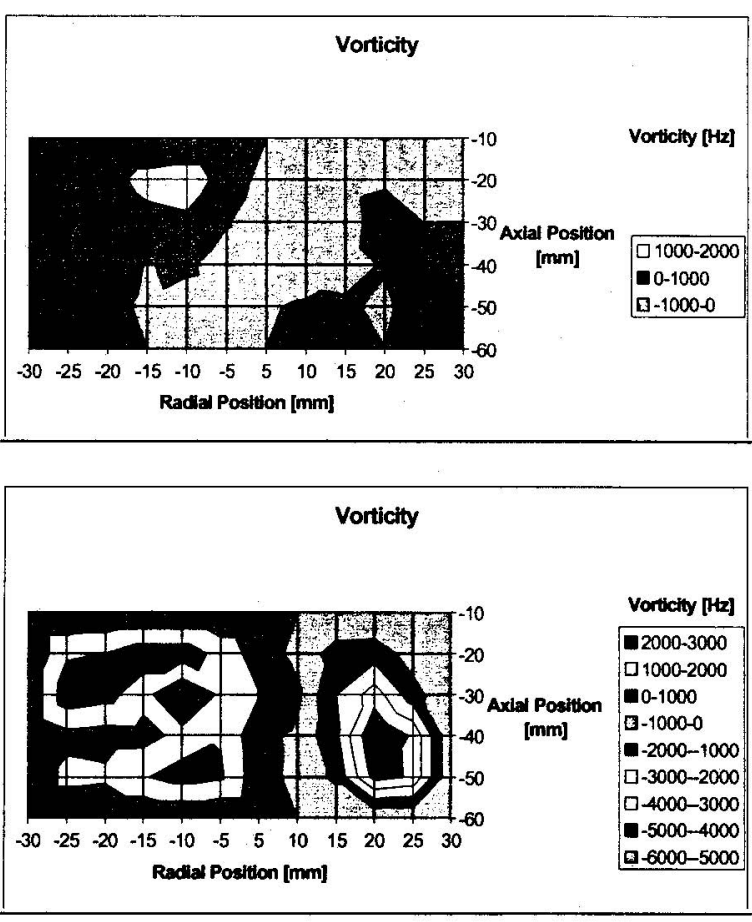

Figure 4. Vortex Structure in the Plane under the Valves.

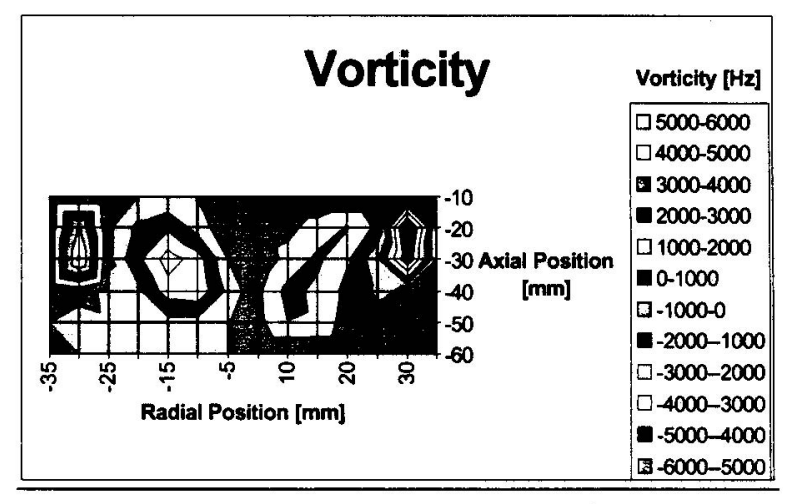

Figure 5. Vortex Structure Showing Symmetry

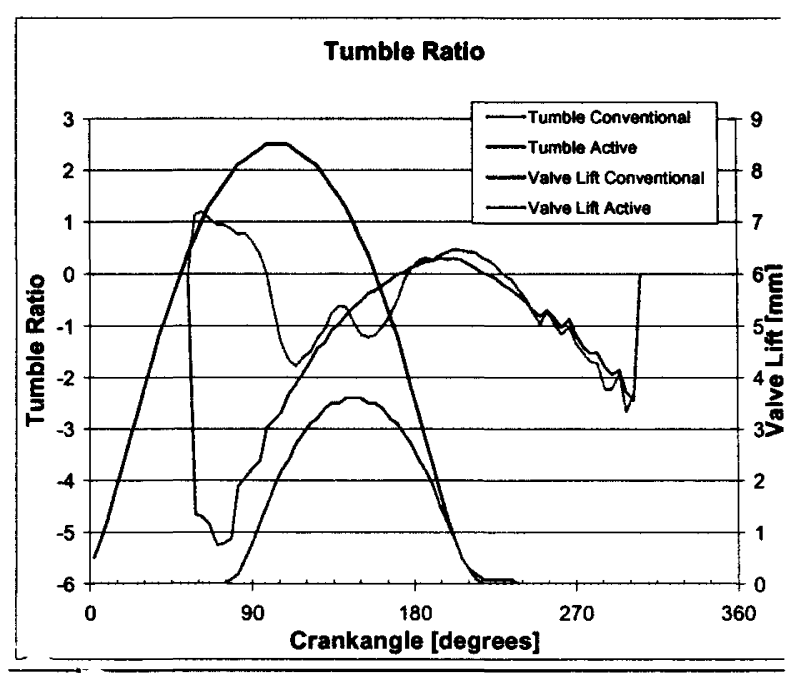

Figure 6. Tumble Ratios 


\section{TURBULENCE KINETIC ENERGY}

Some care must be taken in interpreting these results, as the RMS level is a combination of both the turbulent fluctuations in the flow and also the result of any cycle to cycle variability, although found to be small.

In general, the TKE levels are higher for the modified cams and is probably due to the high piston speed at the late inlet valve opening. Of particular note, are the distributions in the symmetry plane, where the highest levels are seen on the inlet valve side of the cylinder for the modified cams, whereas for the conventional cams they are more centrally located. In all instances the highest levels are seen closest to the cylinder head. An example of the contour plots, in the symmetry plane at 114 degrees after top dead centre are shown in figure 7 . The upper plot is for the conventional cams, and these plots highlight the points made in the above discussion.

It is believed that in the case of the modified cams the combination of small high-speed vortices, combined with high TKE levels produced would lead to a more thorough mixing in the cylinder. What can not be ascertained from these measurements is whether this mixing would include the trapped gases or not, as there is no way to differentiate between this gas and the fresh charge being introduced.

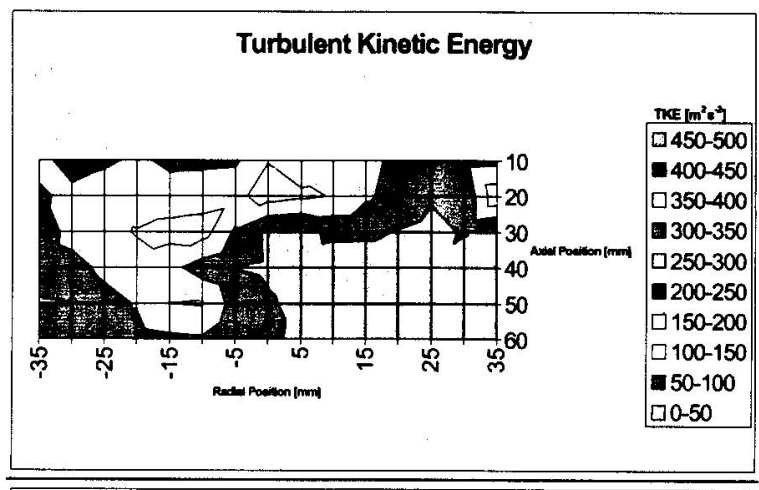

Turbulent Kinetic Energy

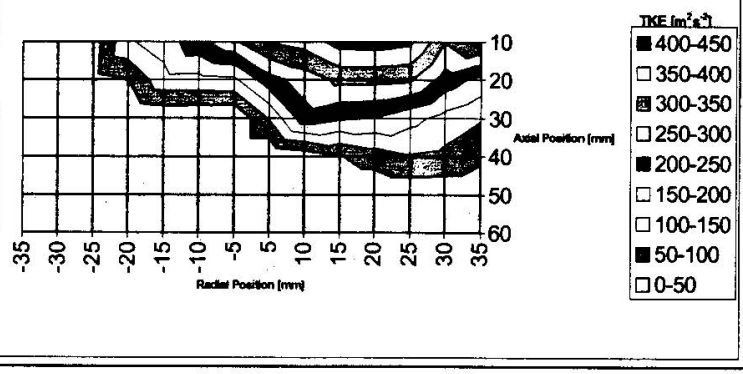

Figure 7. TKE Contours in the Symmetry Plane

\section{CHARGE HOMOGENEITY}

The experiment with the AVT engine to examine any differences between running with $3.6 \mathrm{~mm}$ valve lifts rather than $6 \mathrm{~mm}$, did show one difference between the two engine data sets. The combustion for the 3.6 $\mathrm{mm}$ valve lift occurred later in the engine cycle, as shown by the cylinder pressure traces in figure 8 .
This figure also shows that the lower valve lift had very little effect on the peak compression pressures seen in-cylinder before combustion. It is believed that the valve timing is the important criteria as regards CAl operation. This view is supported in so much as both the AVT and optical engines have been successfully run in CAI mode with the $3.6 \mathrm{~mm}$ valve lift.

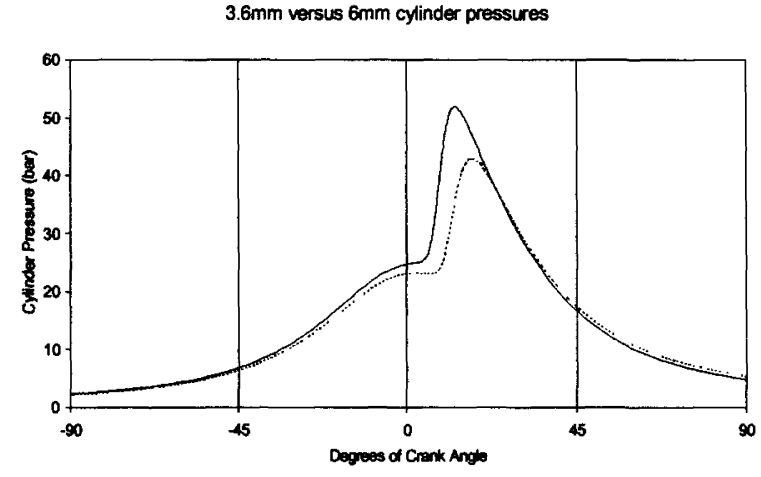

Figure 8. Comparison of in-cylinder pressure traces for $3.6 \mathrm{~mm}$ (dotted) and $6 \mathrm{~mm}$ (solid) valve lift profiles

Similar delays in the point of ignition have been found by running the AVT engine with other valve timing strategies. CAl has also be initiated by bringing exhaust back into the cylinder by opening both exhaust valves during the early inlet stroke. By changing this strategy to opening just one exhaust valve it was noted that the combustion also occurred later in the engine.

The study, presented here, of the flow fields generated in cylinder suggests that a possible cause for the later combustion could be that a less homogeneous mixture between the fresh charge and the induced exhaust gases is responsible. This partial mixing effect with the $3.6 \mathrm{~mm}$ valve lift is seen by the fresh air charge unable to penetrate deep into the cylinder, and therefore does not produce sufficient mixing with the residual exhaust gases.

\section{CONCLUSION}

An analysis of the flow fields observed in this comparison as generated by valve timing strategies for conventional spark ignition and controlled autoignition leads to the following:

- The vorticity of the flow field generated by the CAl cams shows evidence of more and smaller vortices. In the later part of the cycle these vortices have a much greater rotational velocity. This is believed to be due to the much smaller valve lift for these cams causing a greater inlet velocity through the valve gap late in the engine inlet stroke. These vortices persist beyond bottom dead centre, whereas for the conventional cams, the vortices have, to a large extent, diminished by this time. 
- The highest tumble ratios are seen during inlet valve opening, with the conventional cams producing much higher magnitudes. The CAI modified cams produce a second, but lower peak just after maximum valve lift. By bottom dead centre the two tumble ratios tend to a common value, as any influence from the inlet jets diminishes as the valves close.

- The turbulence kinetic energy levels with the CAI cams are higher due to the late valve timing responsible for high inlet velocities late in the inlet stroke. The TKE distributions for the symmetry plane show the highest levels on the inlet valve side of the cylinder, whereas for conventional cams they are more centrally located. In all instances the highest levels are seen closest to the cylinder head.

It is believed that due to the lack of penetration of the fresh charge into the IEGR, the combination of small high-speed vortices with high turbulence kinetic energy levels persisting beyond BDC, as produced by the cam profiles necessary for CAl, do not give thorough charge mixing in the cylinder.

\section{REFERENCES}

1. Lavy at al, Innovative Ultra Low NOx Controlled Auto Ignition Combustion Process for Gasoline Engines, SAE 2000-01-1837.

2. D.Law, J.Allen, D.Kemp and P.Williams, 4-Stroke Active Combustion (Controlled Auto-Ignition) Investigations Using a Single Cylinder Engine with Lotus Active Valve Train (AVT), $21^{\text {st }}$ Century Emissions Technologies Conference, Inst.Mech.Eng., Paper C588/006/2000, London, 4-6 ${ }^{\text {th }}$ December 2000.
3. D.Law, D.Kemp, J.Allen, G.Kirkpatrick and T.Copland, Controlled Combustion in an IC Engine with Fully Variable Valve Train, SAE 2001-01-0251.

4. J.Allen and D.Law, Advanced Combustion Using a Lotus Active Valve Train; Internal Exhaust Gas Recirculation Promoted Auto-Ignition, Proc. IFP International Congress, A New Generation of Engine Combustion Processes for the Future ?, IFP, Paris, 26-27 ${ }^{\text {th }}$ November 2001.

5. H.Ando and K.Kuwahara, A Keynote on Future Combustion Engines, SAE 2001-01-0248.

6. G.Wigley, G.K.Hargrave and J.Heath, A High Power, High Resolution LDAPDA System Applied to Dense Gasoline Direct Injection Sprays, ILASS-Europe, Lisbon, 1998.

7. N.S.Jackson, J.Stokes, M.Sadler, M.R.Heikal, M.Faura and L.Pommler, Correlation of the Combustion Characteristics of Spark Ignition Engines With In-Cylinder Flow Field Analysis Using PIV in a Water Analogy Rig, SAE 971637.

8. G.Pitcher and G.Wigley, LDA Analysis of the Tumble Flow Generated in a Motored 4 Valve Engine, $9^{\text {th }}$ Int.Conf., Laser Anemometry Advances and Applications, Limerick, September 2001.

\section{CONTACT}

G.Wigley@lboro.ac.uk or GPitcher@lotuscars.co.uk 